\title{
Laser information encoded in atomic asymmetrical ionization in few-cycle laser fields
}

\author{
T. Tabe, ${ }^{1}$ N. Ono, ${ }^{1}$ X. M. Tong, ${ }^{1,2, *}$ and N. Toshima ${ }^{1}$ \\ ${ }^{1}$ Institute of Materials Science, Graduate School of Pure and Applied Sciences, University of Tsukuba, 1-1-1 Tennodai, \\ Tsukuba, Ibaraki 305-8573, Japan \\ ${ }^{2}$ Center for Computational Sciences, University of Tsukuba,1-1-1 Tennodai, Tsukuba, Ibaraki 305-8577, Japan
}

(Received 13 June 2011; published 16 August 2011)

\begin{abstract}
The pulse duration and carry-envelope phase (CEP) are two important parameters to characterize a few-cycle intense laser. We systematically study asymmetrical ionization of Ar in few-cycle laser fields with different pulse durations (3-7 fs) and laser intensities $\left(10^{13}-10^{14} \mathrm{~W} / \mathrm{cm}^{2}\right)$ by solving the time-dependent Schrödinger equation. Analyzing the CEP-dependent asymmetry of above-threshold ionization as a function of the photoelectron energy, we find that the qualitative global features of CEP-dependent asymmetry are insensitive to the pulse duration. But the fine structures of the asymmetry are sensitive to the laser pulse duration. Changing Ar to $\mathrm{H}$ atoms, we find that the information encoded in asymmetry is insensitive to target atoms at a moderate laser intensity.
\end{abstract}

DOI: 10.1103/PhysRevA.84.023409

PACS number(s): $32.80 . \mathrm{Rm}, 42.50 . \mathrm{Hz}, 42.65 . \mathrm{Re}$

\section{INTRODUCTION}

Infrared laser interaction with atoms, molecules, and materials is a hot research topic. Most phenomena, such as high-order harmonic generation (HHG) [1], above-threshold ionization (ATI) [2,3], and nonsequential double ionization $[4,5]$, can be explained by the three-step model proposed by Corkum [6]. Laser intensity and wavelength are two parameters that describe laser-material interactions. As does the emergence of few-cycle laser pulses [7], the observed HHG or ATI also depends on the pulse duration and carrier-envelope phase (CEP). By manipulating the CEP, one can tune the time variation of the laser electric field [8] or the outcome of HHG [9] and ATI spectra [10]. The attosecond pulse, a new portable extreme ultraviolet (XUV) light source, can be generated with a stabilized CEP through HHG [11-13]. The attosecond pulse can be used to probe atomic or molecular dynamics [14-16] on a femtosecond or even attosecond time scale. One can also control electron emission [17-19] or electron localization in molecular dissociation $[20,21]$ by tuning the CEP.

Pulse duration and CEP play an important role in few-cycle laser pulse interaction with materials. Unfortunately, it is difficult to measure the CEP directly [22]. The CEP can be retrieved from asymmetrical ionization of atoms in few-cycle laser pulses $[23,24]$. In the experiments, they measured the asymmetrical parameters for high and low energy electrons and retrieved the relative CEP using the fact that the phase of asymmetrical parameters for high and low energy electrons differs by $\pi / 2$. Micheau et al. [25] proposed retrieving the CEP from the photoelectron momentum distribution, especially from the energy peak positions on the right and left sides. For a few-cycle laser pulse, it is also difficult to measure the pulse duration precisely. So far, pulse durations have been retrieved by a frequency-resolved optical gating (FROG) method [26] or spectral phase interferometry for direct electric field reconstruction (SPIDER) method [27,28]. Recently, Sayler et al. [29] proposed retrieving the pulse duration using the asymmetry of ATI spectra. We know that the structure of asymmetrical ionization depends on the laser

\footnotetext{
*tong@ims.tsukuba.ac.jp
}

pulse parameters. Or in other words, the pulse duration and the CEP are encoded in ATI spectra and the question is how to retrieve the information.

In this paper, we studied the asymmetrical ionization of $\mathrm{Ar}$ in few-cycle laser fields with different intensities and pulse durations by solving the time-dependent Schrödinger equation. Our goal is to provide a reliable result for asymmetry of ATI spectra. By comparing the simulated asymmetry of ATI spectra as a function of the CEP and the photoelectron energy with measured ones, one can retrieve the pulse duration and CEP simultaneously.

\section{THEORETICAL METHODS}

Since the detailed numerical method has been published in our previous papers [30,31], we give a brief description of the working equations. All the dynamical information on atoms in an intense laser field is contained in a time-dependent wave function, which can be obtained from the following timeintegral equation as (atomic units $\hbar=m=e=1$ are used through the paper unless stated otherwise)

$\Psi(t)=-i \int_{-\infty}^{t} e^{-i \int_{t^{\prime}}^{t} H\left(t^{\prime \prime}\right) d t^{\prime \prime}} V_{\mathrm{ext}}\left(t^{\prime}\right) e^{-i H_{0} t^{\prime}} \Psi_{0} d t^{\prime}+e^{-i H_{0} t} \Psi_{0}$.

Here $\Psi_{0}$ is the ground-state wave function, $H_{0}$ is the laserfield-free atomic Hamiltonian, $V_{\mathrm{ext}}(t)$ is the electron laserfield interaction, and $H(t)=H_{0}+V_{\text {ext }}(t)$. In the single-activeelectron approximation, the atomic Hamiltonian is written as $H_{0}=-\nabla^{2} / 2+V(r)$, with $V(r)$ a model potential [32]. $V_{\text {ext }}(t)$ is read as

$$
V_{\mathrm{ext}}(t)=-z E_{0} e^{-2 \ln 2 t^{2} / \tau^{2}} \cos (\omega t+\delta),
$$

where $z$ is the electron $z$ coordinate and $E_{0}, \tau, \omega$, and $\delta$ are the peak electric field, the full width at half-maximum (FWHM) pulse duration, the laser-field frequency, and the CEP of the applied laser, respectively. We assumed that the laser field is polarized along the $z$ direction and has a Gausssian pulse envelope. To avoid any confusion, we defined the right side along the positive laser-field direction and the left side along 
the negative-field direction. We discretized the space in the speudospectral grid [33] and propagated the time-dependent wave function by the split-operator method in the energy representation [31]. Numerical simulations were performed in a finite box. To avoid unphysical reflection at the boundary, we separated the space into two regions. When the time-dependent wave function in space reached the outer region, we projected the outer-region wave function into momentum space (Volkov state) and removed it from the wave function in real space. The procedure allows us to keep all phase information for a long time propagation without reflection from the boundary. For a very short pulse, we can directly project the wave function on the laser-field-free atomic continuum eigenstates to get the ATI spectra. We calibrated our ATI spectra with the ATI spectra obtained in this way and they are in good agreement. The drawback of this direct projection is that we have to use a very large box to make sure the photoelectron does not reach the boundary when the laser pulse is over. Thus, it cannot be used for a relatively longer time propagation. In the simulation, we factored out the dynamics irrelevant term [the second term on the right side of Eq. (1)] to improve the numerical accuracy. When the laser pulse is over, the wave function in momentum space is expressed as

$$
\Psi_{\mathbf{p}}(\infty)=\int C(\mathbf{p}) \frac{e^{i \mathbf{p} \cdot \mathbf{r}}}{(2 \pi)^{3 / 2}} d^{3} \mathbf{p},
$$

which contains all the photoelectron dynamic information. The final momentum distribution is read as

$$
\frac{d P(\mathbf{p})}{d E d \Omega}=\sqrt{2 E}|C(\mathbf{p})|^{2},
$$
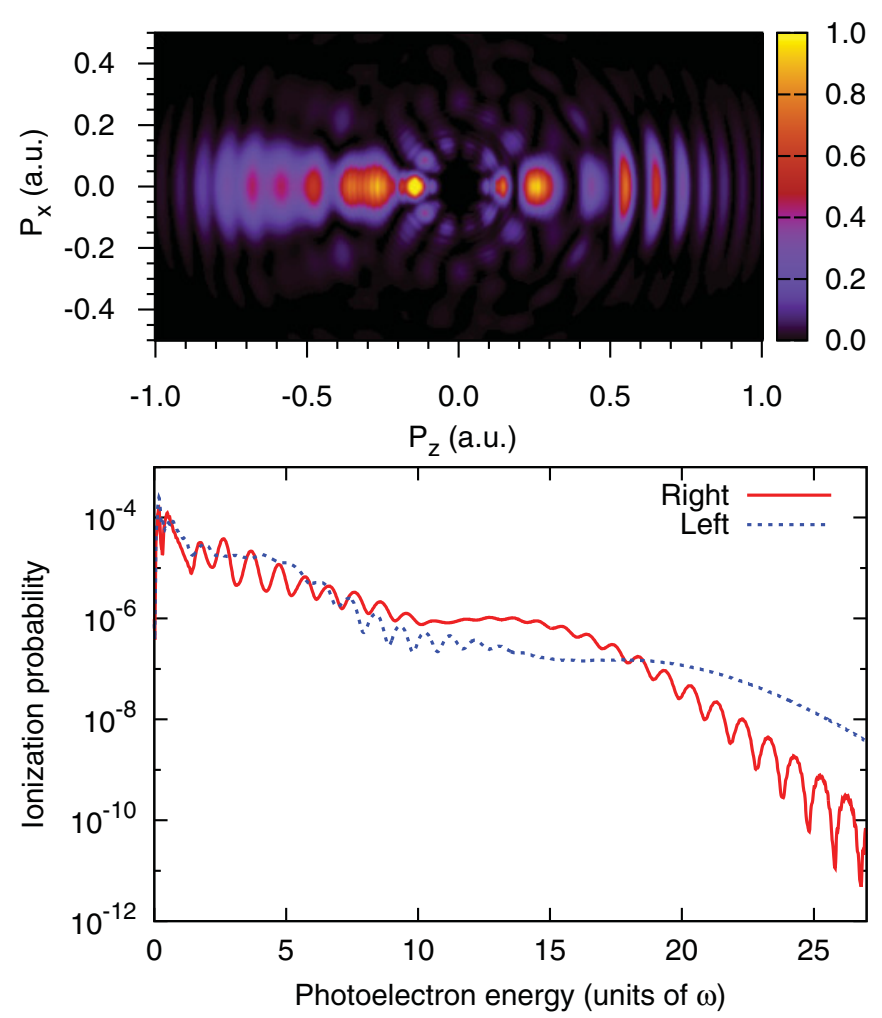

FIG. 1. (Color online) Momentum distribution (upper panel) and ATI spectra on the right and left sides (lower panel) for Ar with a laser intensity of $5 \times 10^{14} \mathrm{~W} / \mathrm{cm}^{2}$, a pulse duration of $5 \mathrm{fs}$, and $\delta=0$. with $E$ the photoelectron energy associated with p. From the momentum distribution, we can obtain the total ionization probability by integrating over the photoelectron energy and emission angle, and the ATI spectra by integrating over the emission angle $\Omega$. In this work we focused on asymmetrical ionization by analyzing the probability of photoelectrons on the right and left sides as

$$
\begin{aligned}
& P_{R}(E)=\int_{R} \frac{d P(\mathbf{p})}{d E d \Omega} d \Omega, \\
& P_{L}(E)=\int_{L} \frac{d P(\mathbf{p})}{d E d \Omega} d \Omega,
\end{aligned}
$$

where $R(L)$ denotes the integration over the right (left) halfsphere. The asymmetry $A(E)$ for a given photoelectron energy $E$ is defined as

$$
A(E)=\frac{P_{L}(E)-P_{R}(E)}{P_{L}(E)+P_{R}(E)} .
$$

\section{RESULTS AND DISCUSSION}

Based on the above theoretical method, we studied asymmetrical ionization of $\mathrm{Ar}$ in few-cycle laser fields. The laser intensities are from $10^{13}$ to $10^{14} \mathrm{~W} / \mathrm{cm}^{2}$ with a laser wavelength of $800 \mathrm{~nm}$ and the pulse durations are 3, 5, and $7 \mathrm{fs}$, respectively. Numerical convergency has been checked by
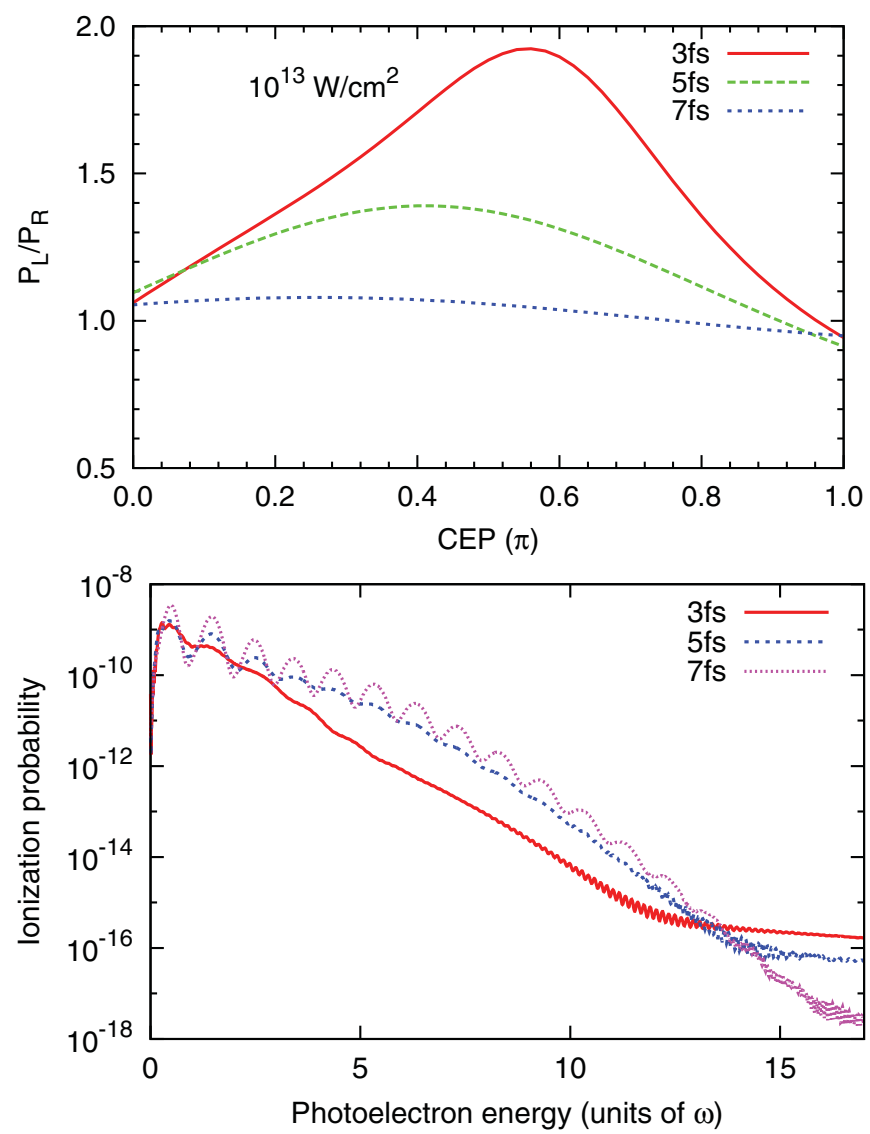

FIG. 2. (Color online) The ratios of total ionization probabilities to the left and right sides (upper panel) and the ATI spectra at $\delta=0$ (lower panel) of Ar in few-cycle laser pulses with a laser intensity of $10^{13} \mathrm{~W} / \mathrm{cm}^{2}$. 
varying the number of grid points, maximum partial wave, and box size in real space. The present results are calculated with a box size of $400 a_{0}$ ( $a_{0}$ denotes the Bohr radius), 72 partial waves, and 1536 radial grid points. When Ar atoms are placed in a linear polarized laser field, the electron density of the valence shell $3 p$ with the magnetic quantum number $m=0$ is aligned parallel to the laser field and it has a ionization probability higher than that for $3 p$ with $|m|=1$ [34]. Thus, we focused on the ionization of $\operatorname{Ar} 3 p(m=0)$ without considering the contribution from $3 p$ with $|m|=1$.

Figure 1 shows the momentum distribution (upper panel) and the ATI spectra on the right and left sides for Ar in a 5-fs laser pulse with an intensity of $5 \times 10^{14} \mathrm{~W} / \mathrm{cm}^{2}$ and $\delta=0$. In the upper panel we see many rings, each of which represents an ATI peak. Due to the nature of few-cycle pulses, the right and left are not symmetric and ATI electrons are mainly emitted along the laser polarization direction. ATI peaks are sharper on the right side than on the left side. Integrating over the right (left) half-sphere, we obtained the ATI spectra on the right
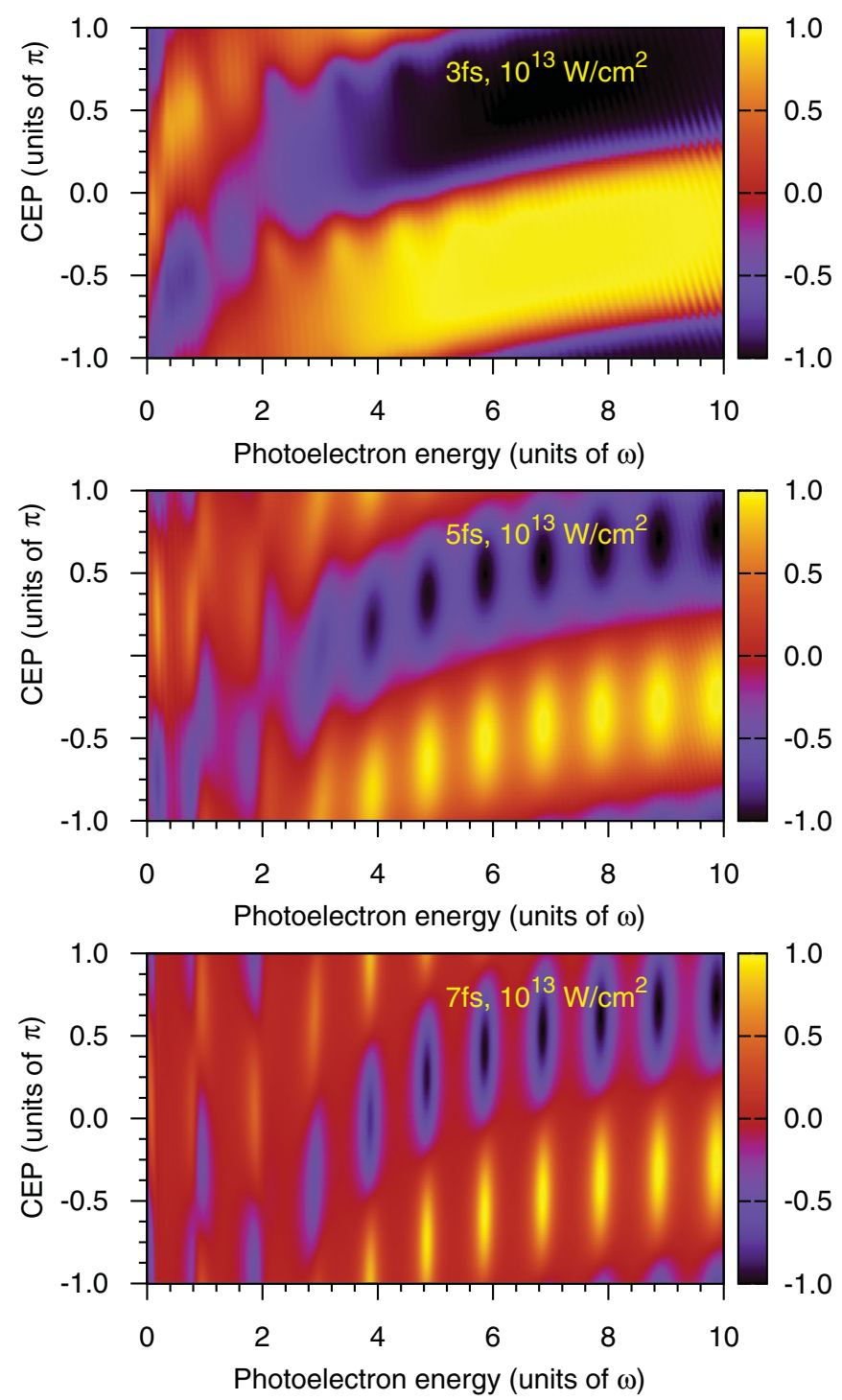

FIG. 3. (Color online) Asymmetry of ATI spectra of Ar at different laser pulse durations with a laser intensity of $10^{13} \mathrm{~W} / \mathrm{cm}^{2}$. (left) side as shown in Fig. 1 (lower panel). For lower energy spectra $(<10 \omega)$, the peaks on the right side are sharper than those on the left side and the ionization probabilities are of comparable magnitudes. Generally speaking, the probability for the left is slightly higher than that for the right. These are consistent with the momentum distribution in the upper panel in Fig. 1. For the middle energy region $(10 \omega-17 \omega)$, there are no sharp ATI peaks and the probability for the right side is higher than that for the left. With the knowledge of the momentum distribution and the ionization probabilities for the right and left sides, we analyze the asymmetrical ionization of $\mathrm{Ar}$ at different laser intensities with three pulse durations.

At the low intensity of $10^{13} \mathrm{~W} / \mathrm{cm}^{2}$, multiphoton ionization is dominant and ATI should weakly depend on the CEP. Figure 2 (upper panel) shows the ratios of the total ionization probabilities for the left and right for three pulse durations.For the shortest pulse duration ( $3 \mathrm{fs}$ ), the maximum ratio is close to 2.0 and the ratio peaks at $\delta=0.56 \pi$. When $\delta>0.5 \pi$, the maximum electric field is on the left side, and when $\delta<0.5 \pi$, the maximum electric field is on the right side. This means that most of the photoelectrons are ejected along the maximum-electric-field direction. The peak positions move below $0.5 \pi$ for longer pulse durations. The maximum ratio decreases dramatically as the laser pulse duration increases and it should approach unity for an infinitely long pulse. To compare to an experiment, we have to know whether or not the ionization probability is measurably high. Figure 2
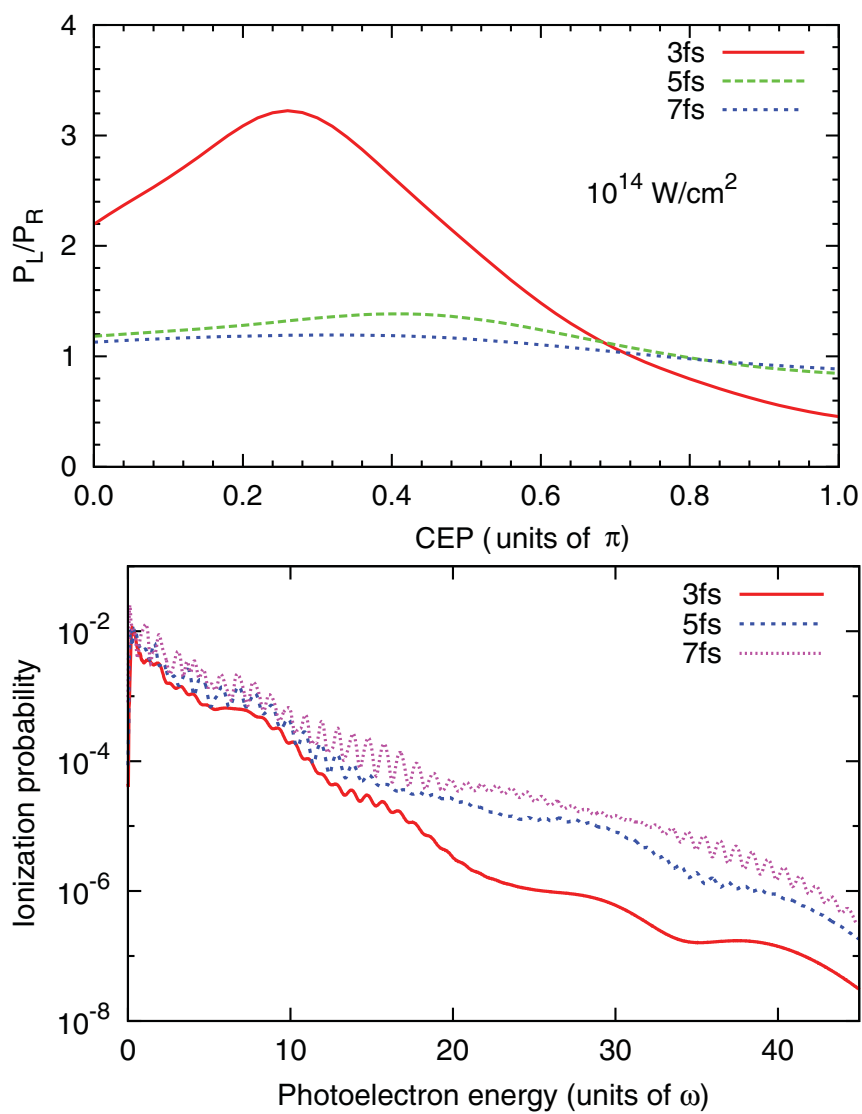

FIG. 4. (Color online) Ratios of total ionization probabilities for the left and right sides (upper panel) and ATI spectra (lower panel) of $\mathrm{Ar}$ in few-cycle laser pulses with a laser intensity of $10^{14} \mathrm{~W} / \mathrm{cm}^{2}$. 
(lower panel) shows the ATI spectra at $\delta=0$ as an example. Ionization probabilities decrease quickly as the photoelectron energy increases and the magnitude does not depend strongly on the pulse duration. For the shortest pulse duration, there are no clear ATI peaks because the laser pulse duration approaches one optical cycle. As the pulse duration increases, ATI peaks appear. Within a 10-photon energy, the ionization probability decreases by six orders of magnitude. With the present technology, the ionization probability at this magnitude should be measurable, although it may not be easy.

Figure 3 shows the asymmetry of ATI probabilities for $\mathrm{Ar}$ in few-cycle laser fields at three pulse durations. For the shortest pulse ( $3 \mathrm{fs}$ ), in the negative-CEP region there is a broad yellow band which moves up as the photoelectron energy increases from $4 \omega$ and it reaches the positive-CEP region. For the lower photoelectron energy, the colored band is mainly located in the positive-CEP region. As indicated in our previous study [30], low-energy electrons come from direct ionization and high-energy electrons come from rescattering
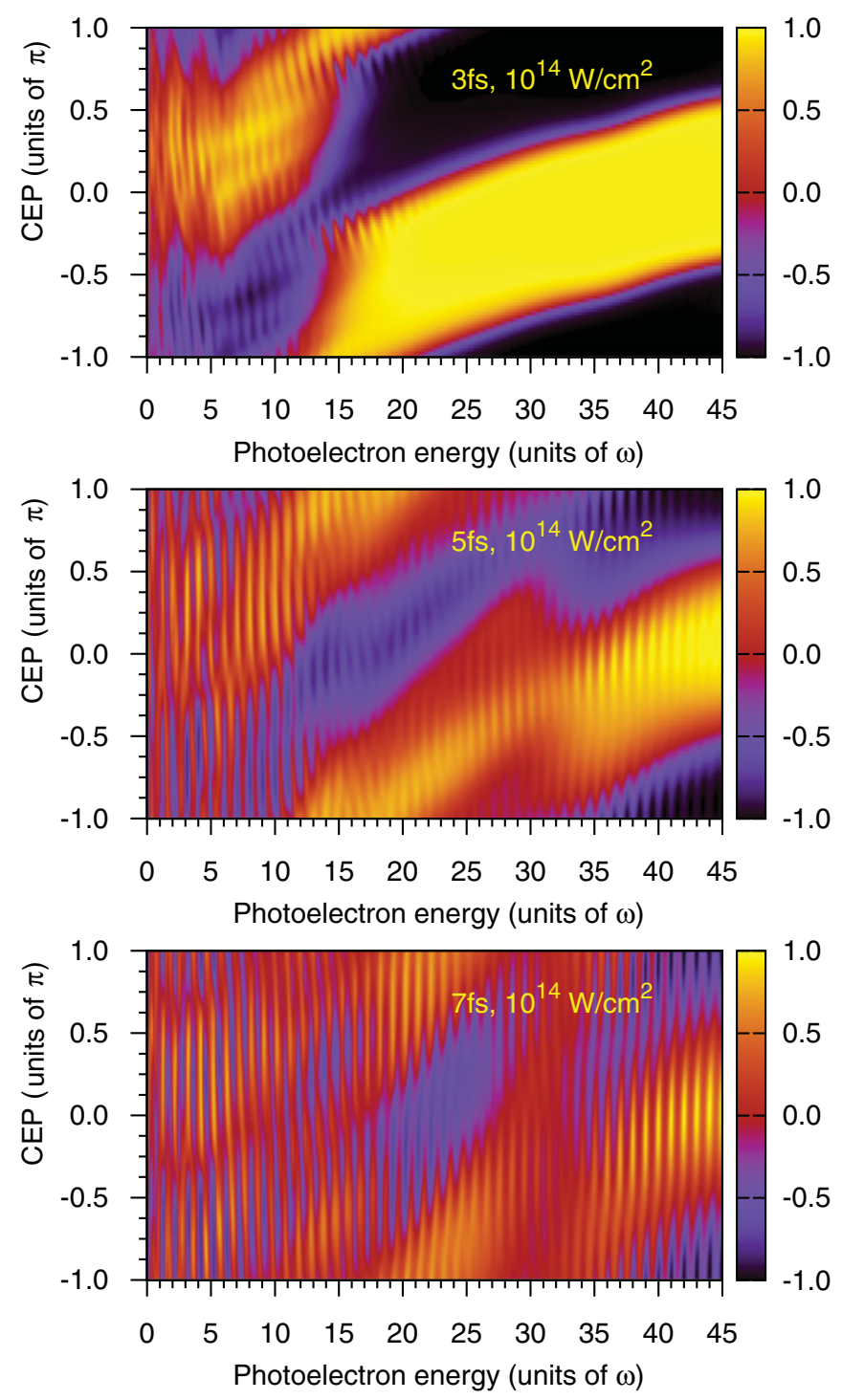

FIG. 5. (Color online) Asymmetry of ATI spectra of Ar at different laser pulse durations with a laser intensity of $10^{14} \mathrm{~W} / \mathrm{cm}^{2}$. electrons, and the relative phase between the two is about $\pi / 2$. Therefore, low-energy and high-energy electrons come from different ionization mechanisms. The yellow color means that the photoelectrons are mainly ejected to the left side. For the longer pulse ( $5 \mathrm{fs}$ ), the qualitative global band structure still exists and is similar to that of the shortest pulse. Differently from the shortest pulse, there are many vertical yellow strips separated by one photon energy. For the longest pulse ( $7 \mathrm{fs}$ ), apart from the global band structure, the vertical yellow stripes are even narrower. By comparing the asymmetry for the three pulse durations, we found that the qualitative global structures are insensitive to pulse duration, while the width of the strips inside the band depends strongly on the pulse duration. The longer the pulse duration is, the narrower the strip width is. If we increase the intensity by 10 times where tunneling ionization is dominant, does the pattern change dramatically?

Figure 4 (upper panel) shows the ratios of the total ionization probabilities for the left and right sides at three pulse durations for the high laser intensity of $10^{14} \mathrm{~W} / \mathrm{cm}^{2}$. For the shortest pulse duration ( $3 \mathrm{fs}$ ), the maximum ratio is close to 3.3, higher than that for the low intensity [see Fig. 2 (upper panel)], and the ratio peaks at $\delta=0.25 \pi$. This means that most of the photoelectrons are ejected against the maximum-electric-field direction. The high maximum ratio for the high intensity and the shift of peak positions (below $0.5 \pi$ for the high intensity and above $0.5 \pi$ for the lower intensity) infer that the ionization mechanisms are different for the high and low intensities. The peak positions move to $0.5 \pi$ for longer pulse durations. The maximum ratio decreases dramatically as the laser pulse duration increases. ATI spectra at $\delta=0$, as
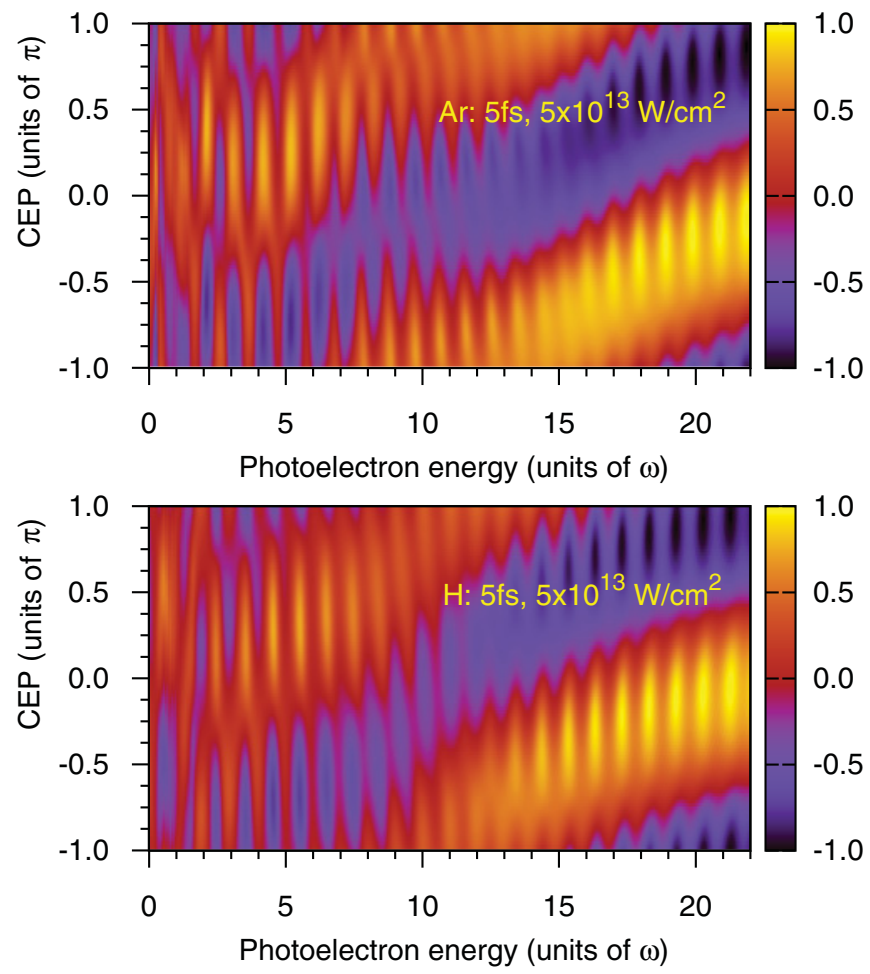

FIG. 6. (Color online) Asymmetry of ATI spectra for Ar (upper panel) and $\mathrm{H}$ (lower panel) in a few-cycle laser field with a laser intensity of $5 \times 10^{13} \mathrm{~W} / \mathrm{cm}^{2}$. 
shown in Fig. 4 (lower panel), extend up to the high-energy end of $40 \omega$ and the ionization probabilities decrease as the photoelectron energy increases. The ionization yields for the high laser intensity are several orders of magnitude larger than those for the low laser intensity.

Figure 5 shows the asymmetry of ATI probabilities of Ar in few-cycle laser fields at three pulse durations for the high laser intensity of $10^{14} \mathrm{~W} / \mathrm{cm}^{2}$. For the shortest pulse ( $3 \mathrm{fs}$ ), similarly to the low-intensity case, at a negative CEP there is a broad yellow band which moves up as the photoelectron energy increases from $15 \omega$. For the longer pulses ( 5 and $7 \mathrm{fs}$ ), the qualitative global band structure still exists and is similar to that of the shortest pulse. Differently from the low-intensity case, there are many structures and the details vary as the pulse duration changes. When tunneling ionization is dominant, photoelectrons at different energies are created by different laser electric peaks and rescattered by the following electric field as we showed in our previous paper [30]. So it is not a surprise that the details of asymmetry are complicated for high intensities. Note that although the global pattern is insensitive to pulse duration, the asymmetry parameter Eq. (7) vanishes in the limit of a long pulse duration. The asymmetry parameter is almost 0 for a pulse duration longer than $10 \mathrm{fs}$.

From the above analysis, we found that the asymmetry of the ATI probabilities for Ar has a global structure which is insensitive to the pulse duration and fine structures which encode the pulse duration information. Does the pattern of asymmetry depend on target atoms? Figure 6 shows a comparison of the asymmetry of ATI spectra of Ar (upper panel) versus H (lower panel).Photoelectrons originate from laser-field ionization of the $3 p$ orbital for Ar and the $1 s$ orbital for $\mathrm{H}$. The ionization probabilities of $\mathrm{H}$ in such laser fields are about 10 times higher than those of Ar due to the lower ionization potential of $\mathrm{H}$. But the general patterns are similar to each other, with a global structure which encodes the CEP information and detailed strips which encode the pulse duration information. Therefore, we conclude that the pulse duration and CEP information encoded in asymmetrical ionization of atoms in a few-cycle laser field is insensitive to the target.

To summarize, we have studied asymmetrical ionization of atoms in a few-cycle laser field and found that the general pattern of asymmetry encodes the pulse duration and CEP information. The CEP can be retrieved from the global structure, while the pulse duration can be retrieved from the fine structures. The information encoded in asymmetry is insensitive to targets when the laser intensity is not too strong. From our study, we suggest a way to retrieve the pulse duration and CEP information using a moderately intense laser field, namely, $5 \times 10^{13} \mathrm{~W} / \mathrm{cm}^{2}$, where multiphoton ionization is dominant.

\section{ACKNOWLEDGMENTS}

This research was supported by a Grand-in-Aid for Scientific Research (C) from the Japan Society for the Promotion of Science.
[1] X. F. Li, A. L'Huillier, M. Ferray, L. A. Lompre, and G. Mainfray, Phys. Rev. A 39, 5751 (1989).

[2] R. R. Freeman, P. H. Bucksbaum, H. Milchberg, S. Darack, D. Schumacher, and M. E. Geusic, Phys. Rev. Lett. 59, 1092 (1987).

[3] W. Becker, A. Lohr, and M. Kleber, J. Phys. B 27, L325 (1994).

[4] B. Walker, B. Sheehy, L. F. DiMauro, P. Agostini, K. J. Schafer, and K. C. Kulander, Phys. Rev. Lett. 73, 1227 (1994).

[5] T. Ishikawa, X. M. Tong, and N. Toshima, Phys. Rev. A 82, 033411 (2010).

[6] P. B. Corkum, Phys. Rev. Lett. 71, 1994 (1993).

[7] A. Poppe, R. Holzwarth, A. Apolonski, G. Tempea, C. Spielmann, T. W. Hansch, and F. Krausz, Appl. Phys. B 72, 373 (2001).

[8] A. Baltuska et al., Nature 421, 611 (2003).

[9] M. Nisoli, G. Sansone, S. Stagira, S. De Silvestri, C. Vozzi, M. Pascolini, L. Poletto, P. Villoresi, and G. Tondello, Phys. Rev. Lett. 91, 213905 (2003).

[10] G. G. Paulus, F. Grasbon, H. Walther, P. Villoresi, M. Nisoli, S. Stagira, E. Priori, and S. D. Silvestri, Nature 414, 182 (2001).

[11] R. Kienberger et al., Nature 427, 817 (2004).

[12] G. Sansone et al., Science 314, 443 (2006).

[13] H. Mashiko, S. Gilbertson, C. Li, S. D. Khan, M. M. Shakya, E. Moon, and Z. Chang, Phys. Rev. Lett. 100, 103906 (2008).

[14] M. Drescher, M. Hentschel, R. Kienberger, M. Uiberacker, V. Yakovlev, A. Scrinizi, T. Westerwalbesloh, U. Kleineberg, U. Heinzmann, and F. Krausz, Nature 419, 803 (2002).
[15] E. Gagnon, P. Ranitovic, X. M. Tong, C. L. Cocke, M. M. Murnane, H. C. Kapteyn, and A. S. Sandhu, Science 317, 1374 (2007).

[16] P. Ranitovic, X. M. Tong, C. W. Hogle, X. Zhou, Y. Liu, N. Toshima, M. M. Murnane, and H. C. Kapteyn, Phys. Rev. Lett. 106, 053002 (2011).

[17] G. G. Paulus, F. Lindner, H. Walther, A. Baltuška, E. Goulielmakis, M. Lezius, and F. Krausz, Phys. Rev. Lett. 91, 253004 (2003).

[18] C. Lemell, X.-M. Tong, F. Krausz, and J. Burgdorfer, Phys. Rev. Lett. 90, 076403 (2003).

[19] A. Apolonski et al., Phys. Rev. Lett. 92, 073902 (2004).

[20] M. F. Kling et al., Science 312, 246 (2006).

[21] X. M. Tong and C. D. Lin, Phys. Rev. Lett. 98, 123002 (2007).

[22] P. Dombi et al., New J. Phys. 6, 39 (2004).

[23] T. Wittmann, B. Horvath, W. Helml, M. G. Schätzel, X. Gu, A. L. Cavalieri, G. G. Paulus, and R. Kienberger, Nature Phys. 5, 357 (2009).

[24] A. M. Sayler, T. Rathje, W. Müller, K. Rühle, R. Kienberger, and G. G. Paulus, Opt. Lett. 36, 1 (2011).

[25] S. Micheau, Z. Chen, T. Morishita, A.-T. Le, and C. D. Lin, J. Phys. B 42, 065402 (2009).

[26] R. Trebino, K. W. DeLong, D. N. Fittinghoff, J. N. Sweetser, M. A. Krumbugel, B. A. Richman, and D. J. Kane, Rev. Sci. Instrum. 68, 3277 (1997).

[27] C. Iaconis and I. Walmsley, Opt. Lett. 23, 792 (1998). 
[28] G. Stibenz and G. Steinmeyer, Rev. Sci. Instrum. 77, 073105 (2006).

[29] A. M. Sayler, T. Rathje, W. Müller, C. Kürbis, K. Rühle, G. Stibenz, and G. G. Paulus, Opt. Express 19, 4464 (2011).

[30] X. M. Tong, K. Hino, and N. Toshima, Phys. Rev. A 74, 031405 (2006).
[31] X. M. Tong and S. I. Chu, Chem. Phys. 217, 119 (1997).

[32] X. M. Tong and C. D. Lin, J. Phys. B 38, 2593 (2005).

[33] J. Wang, S. I. Chu, and C. Laughlin, Phys. Rev. A 50, 3208 (1994).

[34] X. M. Tong, Z. X. Zhao, and C. D. Lin, Phys. Rev. A 66, 033402 (2002). 